\title{
A Method of Pitch Error Compensation Based on HNC-818B CNC System
}

$$
\text { Min LUO }{ }^{a_{1} *} \text {, Hui-ling } \mathrm{LI}^{\mathrm{b}} \text {, Bin MA }{ }^{\mathrm{c}} \text {, Zhi-chu CHEN }{ }^{\mathrm{d}}
$$

School of Electrical \& Information Engineering, Hubei University of Automotive Technology, Shiyan, Hubei, 442002, PR China

ac1636@126.com, b105548927@qq.com,'272345027@qq.com, ${ }^{\mathrm{a}} 15424192 @ q q . c o m$

*Corresponding author

\section{Keywords: Ball Screw; Pitch Error; Positioning Accuracy; Laser Interferometer}

\begin{abstract}
The linear motion of CNC machine is generally converted from rotary motion of servo motor through a ball screw nut pair. As most CNC machine is controlled by semi-closed loop system, ball screw nut pitch error is among the main factors influencing machine positioning accuracy. A method is introduced in this article for CNC linear axis pitch error measurement and compensation by using RENISHAW XL30 laser interferometer. Experiments based on HNC-818B vertical machining center show that bidirectional pitch error compensation for ball screw drive mechanism applying this method can greatly improve the machine positioning precision.
\end{abstract}

\section{Introduction}

At present, the linear motion of CNC machine tool is generally converted from rotary motion of servo motor through a ball screw nut pair. As most CNC machine tools is controlled by semi-closed loop system, when the geometric precision of the machine is guaranteed, the machine axes backlash and ball screw nut pitch error are among the main factors influencing machine positioning accuracy and repetitive positioning accuracy. The compensation for pitch error of the ball screw nut pair can greatly improve the machine tool precision, so as to improve machining accuracy. There are two kinds of special parameter for axis error compensation in machine control systems.

\section{Pitch Error and Compensation}

On one hand, due to the impact of the machining precision and processing conditions change, the ball screw pitch error exists; on the other hand, if screw and nut failed to preload to the desired state during installation, the machine moving parts may not reach a predetermined position. This error is often referred to the pitch error as well.

Pitch error compensation can be divided into two ways: real-time dynamic compensation and static compensation.

Real-time dynamic compensation, also known as online compensation, is a method in which a real-time position detection system is used to measure and thus numerically control the machine tool axes positioning. This method of compensation can significantly improve the positioning accuracy of the machine, but the machine costs will increase accordingly due to the higher system requirements for the machine.

Static homogenized compensation is implemented as the following: pitch error compensation value is stored in the CNC system parameter table in advance; after the compensation values were utilized, the CNC control system will automatically achieve pitch error compensation by superimposing the value on the interpolation command.

\section{Error measurement system using Laser Interferometer}

XL30 laser Interferometer includes optics lens group and electronic circuits, frequency stabilization, subdivision and fringe counting of the laser beam. The lens for measuring linear positioning accuracy includes a beam splitter and two linear reflectors. A reference beam is generated through interference lens which is composed by the splitter and one reflector, a 
measurement beam is generated by another reflector, as shown in Fig.1.

During linear measurement, a lens remains stationary, and another lens moves. Then the positioning errors are achieved by comparing the optical path difference between the measurement beam and the reference beam.

Typically, reflector is set to be movable part, while the interference lens is set to be the stationary one. Both can be reversed, but the maximum measuring distance will be shortened. Therefore, the linear interference lens is generally the stationary part when measuring the long stroke, and is inverted to move when measuring the minor stroke for convenience.

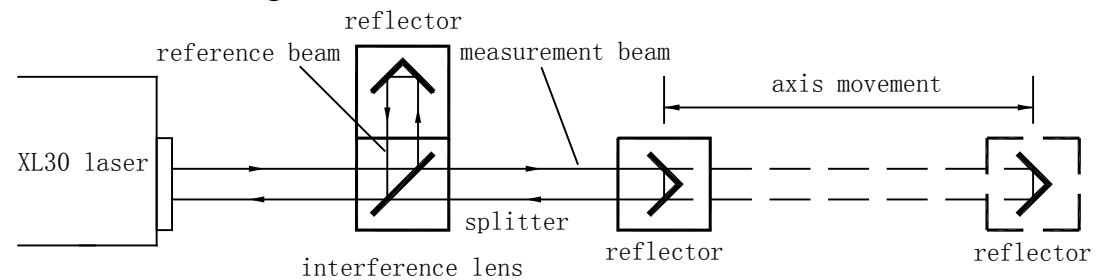

Fig.1 laser interferometer error measurement system

\section{HNC-818B System Parameter Settings}

Here is an example of the X-axis pitch error of measurement and compensation introduced by Huazhong HNC-818B CNC vertical machining center using REN ISHAW company XL30 laser interferometer measurement system.

Firstly,set system parameters related to axis-X pitch error compensation.

300020: pitch error compensation type, is set to 0, no pitch error compensation.

Secondly,set axis-X soft limit, in order to ensure that the laser interferometer beam splitter and mirrors do not collide when measuring.

100006: Positive soft limit coordinate,20.0mm;

100007: Negative soft limit coordinate,-1020.0mm.

\section{Installation and Adjustment of XL30 Laser Interferometer}

(1) Installation of the laser head and linear optics lens

XL30 laser head is fixed and initially leveled by a tripod and holder. The tripod is for height adjustment, while the holder charges for horizontally pan, the torsion angle adjustment and tilt angle adjustment.

Linear interference lens is fixed to the table, and is placed in the optical path between the reflector and the laser head XL30. The reflector is fixed at the end face of the spindle, as shown in Fig.2.

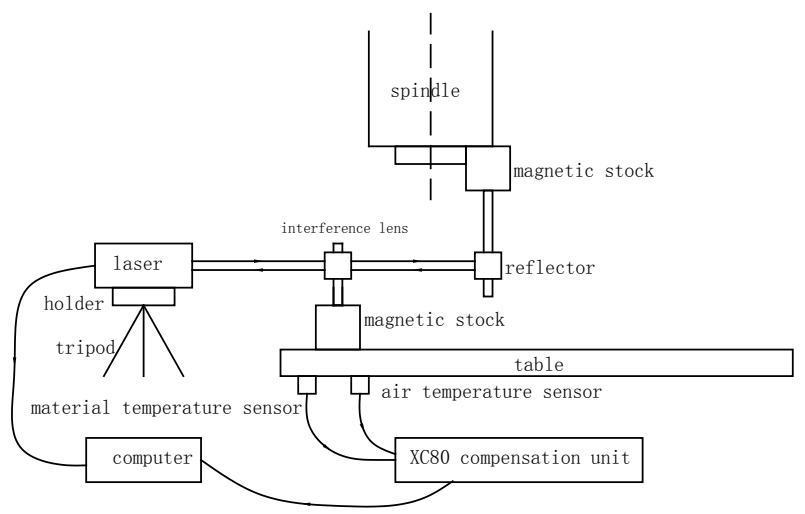

Fig.2 Installation of XL30 Laser Interferometer

(2) Laser Beam Collimation by simply Using Light Target

Using a light target, make the beam hit the white light target on the interference lens in the axis full range. 
Step 1: horizontal adjustment of the beam. Push along the axis direction of movement the laser interferometer group away from the laser head, until the light beam in the horizontal direction can be seen beginning to leave the target. Stop moving when only half of the beam is still hitting the white point, shown in Fig. 3 (a). Adjust the torsion angle of the laser head to make white light beam traverses the target by using a small knob at the left rear of the holder. Continue to move the beam in the opposite direction until it is offset by the same distance from the center, as shown in Fig. 3 (b).

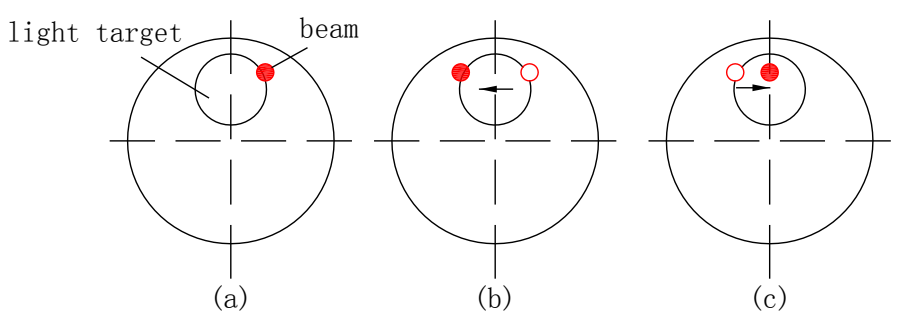

Fig.3 light beam horizontal adjustment

Step 2: using the large knob on the left middle side of the holder, translate the laser head until the beam returns to the horizontal center line of the optical target, as shown in Fig.3 (c).

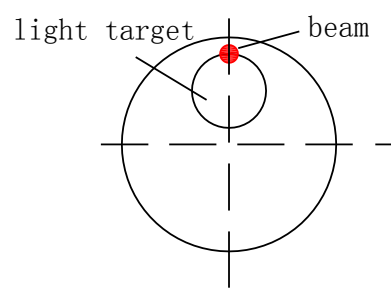

(a)

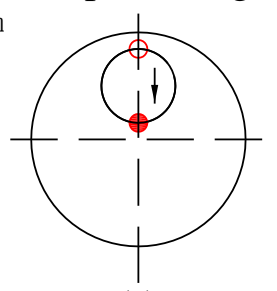

(b)

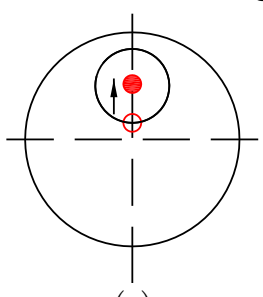

(c)

Fig.4 light beam vertical adjustment

Step 3: vertical adjustment of the beam. Push laser interferometer lens away from the laser head along the movement axis, until the beam starts to leave the target in the vertical direction. Stop moving when only half of the beam still hits the white point, as shown in Fi.4 (a). The holder then be tilted and adjusted until the beam reaches the same position in the opposite direction from the center, as shown in Fig.4 (b).

Step 4: Use the height-adjustment wheel in the tripod center spindle to move the laser head up and down until the beam hits the center of target again, shown as Fig. 4 (c).

Step 5: repeat steps 1 through 4 until light beam can hit both horizontal centerline and vertical centerline of the light target in full axis stroke.

Step 6: Adjust the mirror and make it keep parallel to each surface of the interference lens. Make sure that the reference beam and the reflected beam overlap.

\section{Programming axis- $\mathrm{X}$ bidirectional positioning error measurement}

Measurements start from X-1000.0, positive feed to the reference point X0.0, 20.0mm every step; when the forward measurements end and backlash is eliminated, after $1.5 \mathrm{~mm}$ overrun, negative measuring begins, feeding $-20.0 \mathrm{~mm}$ each step, with the end of $\mathrm{X}-1000.0$. Measurement program is as follows:

G54 G90 G00 X-1000;

G01 X-1.5 F500;

G04 X1.0;

G01 X1.5; eliminate backlash

M00;

$\# 100=1$;

WHILE [\#100 LE 50]

G91 G01 X20.0 F1000; positive feed 20.0mm every step 
G04 X3.0;

$\# 100=\# 100+1$;

ENDWHILE;

G01 X1.5 F500;

G04 X1.0;

G01 X-1.5; eliminate backlash

WHILE [\#100 LE 50]

G91 G01 X-20.0 F1000; negative feed 20.0mm every step

G04 X3.0;

$\# 100=\# 100+1$;

ENDWHILE;

M30;

\section{Error Measurement and Calculation}

After 5 measurements, the average positioning errors of both the positive and negative $\mathrm{X}$ axis motion are obtained, as shown in Fig. 5.

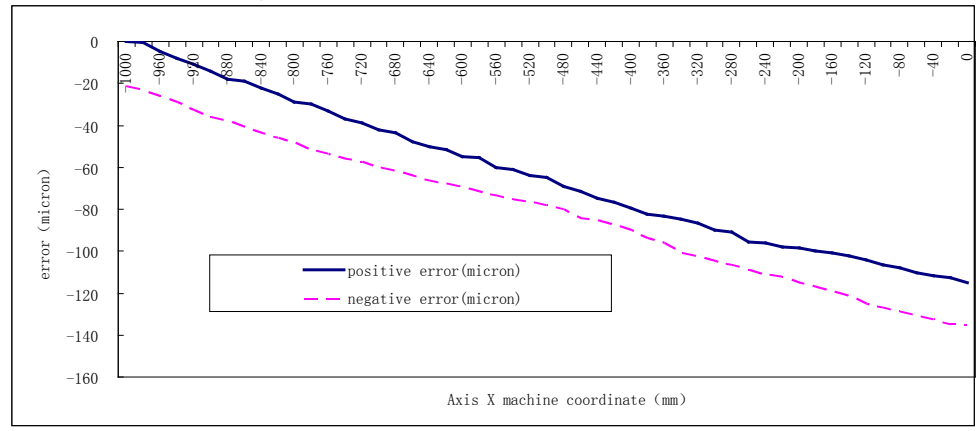

Fig.5 Axis X positive and negative positioning error

\section{Unidirectional Pitch Error Compensation and Effect}

Unidirectional pitch error compensation parameters are set as follow:

300020: Pitch error compensation type. Set to 1, indicating a one-way pitch error compensation.

300021: Pitch error compensation starting point coordinate. Set to $-1000.0 \mathrm{~mm}$.

300022: Number of pitch error compensation points. Set to 51 points.

300023: Pitch error compensation point spacing. Set to 20.0mm

300024: Modulus of pitch error compensation enable. Set to 0, turn-off function.

300025: Pitch error compensation ratio. Set to 1.0.

300026: Pitch error compensation table starting parameter number. Set to 700000.

Parameter700000 700050 are axis-X unidirectional pitch error compensation data: $0.011,0.012,0.015, \ldots, 0.124,0.125$.

The results of unidirectional pitch error compensation are shown as Fig. 6. Both the axis-X positive and negative motion compensations are less effective. 


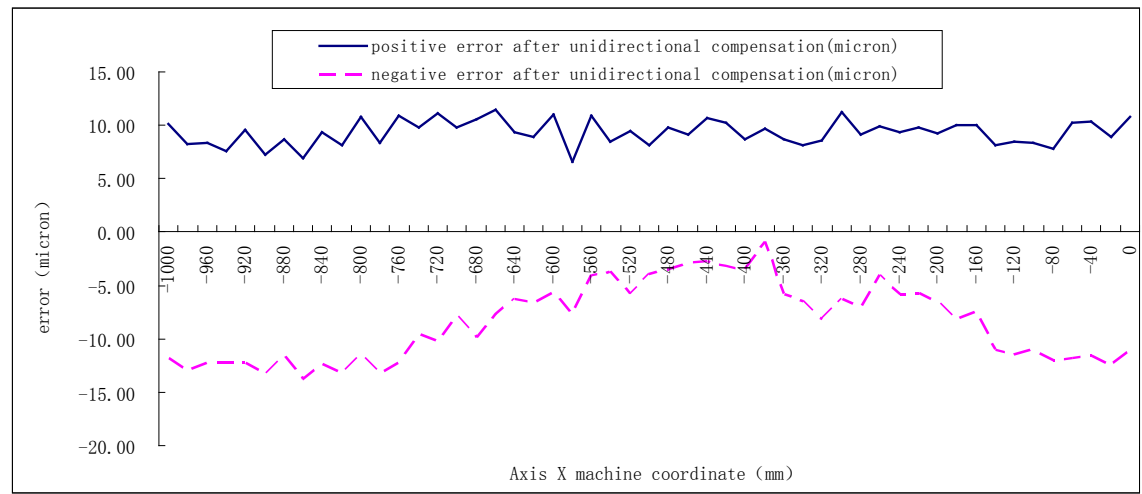

Fig.6 positioning error after unidirectional pitch error compensation

\section{Bidirectional Pitch Error Compensation and Effect}

Bidirectional pitch error compensation parameters are set as follow:

300020: Set to 2, indicating Bidirectional pitch error compensation.

300021 300025: Set the same value as unidirectional pitch error compensation.

300026: Pitch error compensation table starting parameter number. Set to 700100.

Parameter700100 700150 are axis-X positive direction feed pitch error compensation data:0.000,0.001,0.005,0.008,..,0.112,0.115;Parameter $700151 \sim 700200$ are axis-X negative direction feed pitch error compensation data:0.021,0.023,0.026,0.029, ..,0.135,0.135.

The results of bidirectional pitch error compensation are shown as Fig. 6. The compensations work well for both forward and backward motion along the $\mathrm{X}$ axis, with the former positioning error basically within \pm 2 microns, the latter within \pm 3 microns.

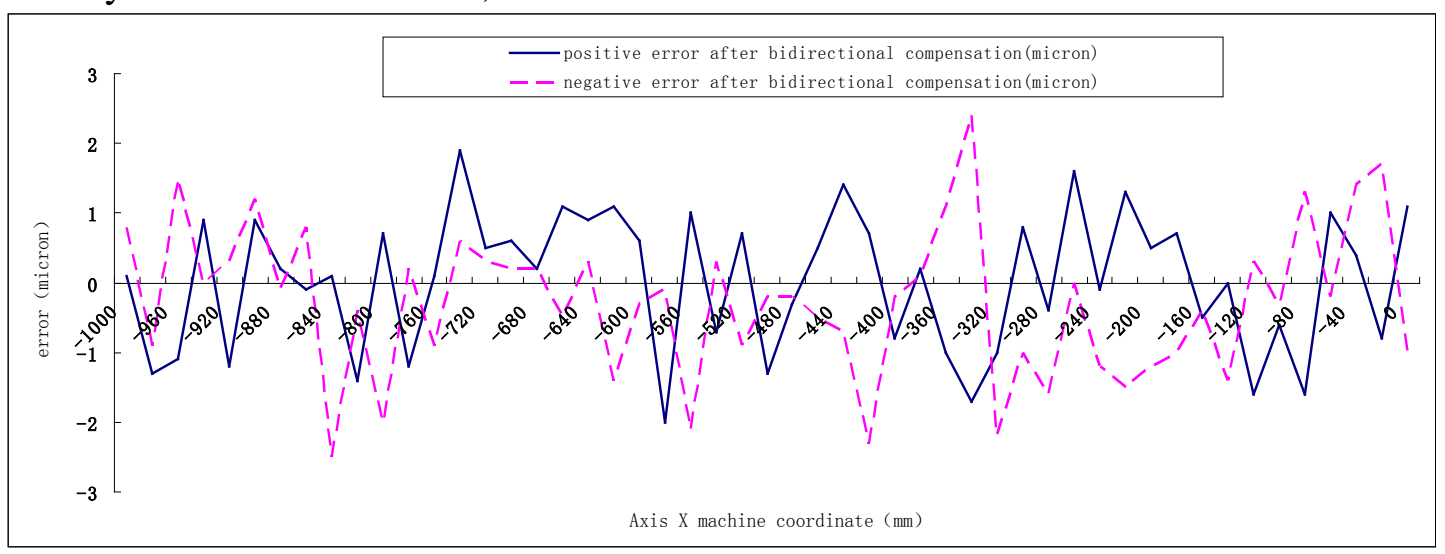

Fig.7 positioning error after bidirectional pitch error compensation

\section{Conclusion}

The basic principle of pitch error compensation lies in the following two facts. First, through repeatedly measuring a series of axis coordinate positions in the effective stroke using high-precision measurement system for CNC machine tools, the average difference between the command position and the actual position of the detection can be calculated. Second, after the error value is inputted into NC system, the compensation can then be automatically implemented in the process to compensation points on the axis.

For a semi-closed-loop controlled CNC machine tools, when the ball screw nut pairs wear over some time or their processing precision and fitting accuracy are not enough at the beginning, pitch error compensation is necessary in order to overcome the ball screw motion accuracy and thus improve processing quality of parts. Unidirectional pitch error compensation cannot meet error compensation consistency requirements for the positive and negative motion of the coordinate axis of CNC machine tools, so the bidirectional pitch error compensation is needed 


\section{Reference}

[1] YUAN Lin,LI Hai-bin,YANG Jing. NC Machine Pitch Error Analysis and Compensation. Aeronautical Manufacturing Technology,2011,(8):90 93

[2] LI Ji-zhong. The Compensation and Analysis of Pitch Error for NC Machining Tools. Modular Machine Tool \& Automatic Manufacturing Technique,2010,(2):98 101

[3] NIU Xiao-tie,CHEN Jin-ying. Accuracy Measurement and Error Compensation of BV75 Vertical Machining Center. Coal Mine Machinery,2013,34(8):131 133

[4] ZHANG Xin. Study on Pitch Error Compensation of HNC-818B CNC Rotary Table. Machine Tool \& Hydraulics,2014,42(2):112 114+130

[5] CAO Yong-jie,WAN Jun,FU Jian-zhong,ZHANG Xiao-san. Errors Analysis of Laser Interferometer During the Linear Positioning Accuracy Measurement in Machine Tools. Machine Tool \& Hydraulics,2007,35(4):163 164+183

[6] CHEN Yong,WANG Qing-chun,XU Wei. The Compensation of Pitch Error for XK714/1 CNC Milling Machine. Machine Tool \& Hydraulics,2012,40(8):24 26

[7] WANG Pei-lin. Method and Experiment of Increasing Precision NC Milling Machine Based on Software Compensation. Mechanical Engineer,2013,(11):84 86

[8] CHEN Yong,XU Wei. Compensation and reconstruction of pitch error for CNC2190 CNC milling machine. Manufacturing Technology \& Machine Tool,2011,(8):207 209

[9] HUANG Min-han,WEI Zuo-zhi. How to Improve the Efficiency of Thread Pitch Error Compensation of CNC Machine. Metrology \& Measurement Technique,2011,38(11):42 43 